\title{
Building facade optimization for maximizing the incident solar radiation
}

\author{
Kyane Bomfim ${ }^{1}$, Felipe Tavares ${ }^{2}$ \\ ${ }^{1,2}$ Faculty of Architecture - UFBA \\ 1,2 \{kyane.bomfim|felipe.tavares\}@ufba.br
}

\begin{abstract}
The technological breakthrough on photovoltaic facades and the high potential for installing photovoltaic $(P V)$ systems in the city of Salvador are the motivation for this article. This case study explores the feasibility of implementing solar energy technology on a building facade, proposing a design method for optimizing insolation performance by the form-finding process in a parameterized shape. The goal was to generate a parametric design workflow, in which it could be found some facade shapes, generating triangle and quadrilateral supporting grids, leading to better results in the total amount of radiation in comparison to the basic flat facade. In these supporting grids were evaluated also the fitting in the distribution of quadrilateral commercial PV cells, measuring its geometric compatibility. By the results, it could be verified the gains and losses in PV potential in several instances obtained by the form-finding process, as the potentials to consider this in the design of every building.
\end{abstract}

Keywords: Radiation skydome, Shape parameterization, Form-finding, Genetic Algorithm, PV facade

\section{INTRODUCTION}

On the mid-twentieth century Third Industrial Revolution, the capitalist urban-industrial environment emerged and has been intensified until today, triggering the production of machines and competitive technological development (Silva et al. 2016). Consequently, there has been an exponential increase on energy demand, which is the main focus of multiple global discussions for promoting renewable energy, as in the Renewable Energy Policy Network for the 21st Century (R.E.N.21 2018).

The global building energy consumption could increase by $50 \%$ to 2050 , without assertive energy efficiency action (I.E.A. 2016). The use of solar energy in buildings is critically important for reducing expo- sure to global warming impacts and associated environmental costs. In order to mitigate environmental problems, it is possible to integrate active solar technologies into buildings (Probst and Roecker 2012). The Photovoltaic (PV) system is a type of active solar technology. In it, devices made with semiconducting material turn sunlight into electricity, generating a direct current when sunlight hits a PV panel, which will then be converted into alternating current through an inverter.

Photovoltaic panels often have been installed on rooftops, because on horizontal surfaces, they can have an optimal tilt angle based on geo-location. While on vertical facades, this inclination may not be optimal, even though they hold significant potential 
(Freitas 2018). The photovoltaic building facade handle, at the same time, aesthetics and architectural design integration issues; moreover, its principal function, generating electric power from the solar radiation.

The facades solar potential analysis demands computational tools which require empirical validation (Freitas 2018). To simulate solar potential on surfaces, these tools are set with weather data input and varying sky conditions through matrix-based calculations (Tregenza and Waters 1983).

Using Computer Aided Design (CAD) parametric modeling, this study proposes modeling NonUniform Rational Basis Spline (NURBS) surfaces to represent the building facade shape. By form-finding process, a Genetic Algorithm (GA) optimizes this parameterized shape. The GA is adopted as a tool for exploring solutions that designers couldn't imagine. Also, it expands the potential of computational design processes, which is no longer just a tool to act as an aid for it (Martino 2015).

This shape optimization to capture more radiation as possible is the focus of this paper, through simulated tests featuring a building, further described in the Case Study section. This chosen building, currently under construction, owns a structural skeleton but lacks the envelope, cladding, and other architectural elements. The goal is to generate the proper geometric shape for one of its facades, by selecting the optimized solutions among several simulations on surfaces, searching for the maximum solar radiation incidence as possible.

However, the form-finding simulation process through GA can be computationally slow when the coupled simulation that gives the fitness function requires some time consumption. The solar radiation simulation used lasts some seconds to perform its algorithm for each model instance, bringing the need to find some simulation alternative that could run a similar evaluation in time below one second. The use of the Radiation Calla Dome component instead of Radiation Analysis, both components from the Ladybug simulation interface, came to be the chosen alternative for significant computational process improvement.

These simulations generated surface geometries from NURBS isocurves providing changes in the control points willing to find optimized solutions. Consequently, the results are vastly diverse, with single or double curvature surfaces, varying the solar radiation analysis results on each instance. Due to the shape parameterization, the geometry is shaped with more regularity and rhythm and these instances have higher solar photovoltaic potential and newer geometric design, compared to the basic vertical flat facade option.

\section{CASE STUDY}

There are a lot of metropolises that invest in photovoltaic solar energy. China, USA, India, Japan, among other countries from North hemisphere are responsible for leading the global PV market. The last country to reach the Gigawatt (GW) production mark in 2017 was Brazil (ninth), which installed slightly more than 1 GW, bringing the total installed capacity in the country to $1,1 \mathrm{GW}$ (I.E.A 2018). In this context, Salvador, the city in Brazil where the case study is located, has the coordinates $-12.97^{\circ}$ latitude and $-38.51^{\circ}$ longitude and demonstrates a higher potential for photovoltaic system implementation than those other countries listed. Throughout the year, Salvador maintains a higher Global Horizontal Irradiance rate. As from the annual data mapped by World Bank Group (W.B.G. 2016), it reaches an average of $2002 \mathrm{kWh} / \mathrm{m}^{2}$ per year, due to its latitudinal location. It is convenient to raise attention about its potential on photovoltaic energy, promoting it as a renewable source of electric generation in architectural design.

The chosen case study is building of classroom and laboratories, still under construction, is an attachment to the Federal University of Bahia - UFBA's Polytechnic School main building. The school parking lot is located in front of this building southwest facade, as Figure 1 demonstrates. This position benefits solar incidence by casting minimum shadows on the building. Hence this, all of the studies here 
Figure 1

Perspective view of the study area

Figure 2

Solar relative position angles presented intent to simulate the proper shape for a photovoltaic facade, for the building new southwest facade, through parametric mathematical processes generating solutions of better performance geometries to optimize catching the solar radiation incidence.

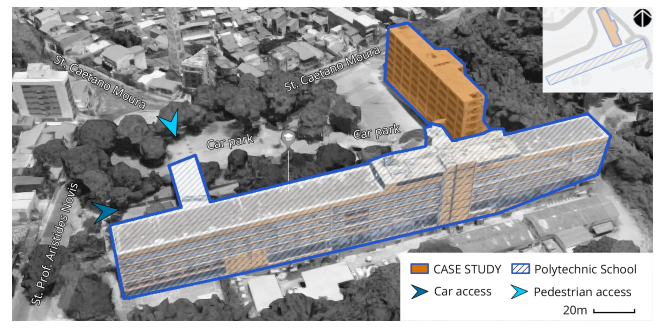

This study brings out different types of curves for generating complex surfaces that allow a higher intensity of incident solar radiation, assuming a basic flat facade as an initial comparative reference. This generative process is explained in the following topic, gathering data and methods.

\section{MATERIALS AND METHODS}

The simulations were performed on an Intel(R) Core (TM) i7-8700K CPU @ 3.70GHz with 64GB RAM and a solid-state disk. The open source environmental plugin Ladybug (Sadeguipour and Pak 2013), upon the Climate-Based Daylight Modeling (CBDM) (Ashdown 2004) considering the Radiance (Ward 1985) as the computing engine for calculating illuminance with Daylight Coefficients. All these applications were used within Rhinoceros/Grasshopper workflow, enabling the calculation of illuminances considering variable annual sky conditions.

The illuminance at a measurement point in the patch surface of the skydome can be calculated to estimate the brightness/luminance of the sky. An annual daylight matrix with 145 patches, the annual Perez Sky Model, creates the sky patch vectors, from an EnergyPlus Weather (EPW) file meteorological data related to a specific city. This weather file contains hourly values of its direct normal and dif- fuse horizontal irradiation that is related to each skyvector, which is required for obtaining the total radiation measuring on a given surface.

The evaluation of the amount of radiation reaching a given surface is performed considering solar position angles relations and calculations regarding local weather database values. Also, it is assumed the 3D sun-path diagram and all its direct beam radiation (sunbeam) vectors, containing the radiation intensity to be related to the given surface normal vector. When the direct solar beam is perpendicular to a surface, it is known that is the best situation to achieve a higher rate of radiant energy upon this surface, expressed in $\mathrm{W} / \mathrm{m}^{2}$ (Sarbu and Sebarchievici 2017). This information was considered in the proposed design method to optimize the incident radiation on a photovoltaic facade shape.

\section{Solar radiation geometry analysis}

Each sunbeam vector from a 3D sun-path diagram (Figure 2 ) is related to a corresponding specific radiation value calculated by the cumulative sky method (Robinson and Stone 2004). And to work with a surface with any inclination and orientation, it is necessary to obtain its normal vectors for computing the angles between it and the sunbeam vectors generated.

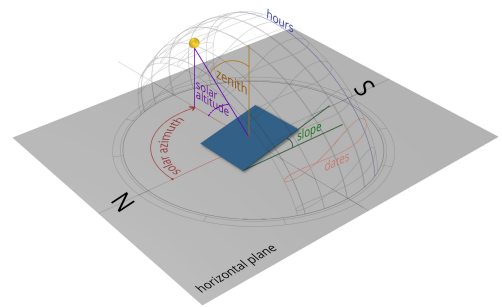

Consequently, the solar radiation input is different for each geographic location, as well, the sunbeams vectors will have different interactions with the normal vectors of the surfaces to be evaluated. As shown in Figure 2, the sun position is related to the North and the zenith angle, as the blue surface represents any tilt plane surface, in which can be a facade surface 
with its angle against horizontal plane close to $90 \mathrm{de}-$ grees.

To represent this sun-path in the $3 \mathrm{D}$ model is necessary to inform as input the North direction and, for this study, it was associated with the global y-axis. The center of those dome models was chosen to be positioned at the building floor plan centroid.

The method of calculating radiation on surfaces combines normal vectors of the surface to be evaluated and sunbeam vectors from the 3D sun-path diagram. Consequently, each sun position in this diagram has a vector pointing to the center of the dome model, in which the amount of incident radiation on an evaluated surface is positioned.

Through the Ladybug simulation interfaces, the radiation calculation through its Radiation Analysis algorithm has proved to be too time-consuming in its computational processing. For each surface with grid-resolution of $1.0 \mathrm{~m}$, it delivers the results in an average time near about $4.45 \mathrm{~s}$.

Nevertheless, to run the facade form-finding process through the GA, the time must be shortened because the GA simulates each genome and within that process, thousands of genomes are generated. It was identified that the GA associated with Calla Dome model provides improvement in the computational time process, being almost $16 \mathrm{x}$ faster than Radiation Analysis.

For this reason, as an alternative to improve processing time and obtaining faster results, the Radiation Calla Dome model was used in the facade shape form-finding process to replace the sunbeam vectors from the sun-path diagram. The computational processing is faster than Radiation simulation since it is not comparing with all 3D sun-path sunbeams vectors, but with a smaller number of vectors from Calla Dome model, increasing the processing speed for each evaluated façade shape model instance.

The Radiation Calla Dome algorithm provides the directions of highest radiation intensity, calculated from the skydome model. In Figure 3 is presented the Radiation Calla Dome over the studied building, showing the Calla Dome radiation intensity vectors. It consists of a dome discretized with $30^{\circ}$ in the horizontal plane base circle and $15^{\circ}$ in the vertical plane. This algorithm model outputs a color gradient representing the radiation intensity, and the vectors for all patches containing a radiation value associated with each direction by vectors.

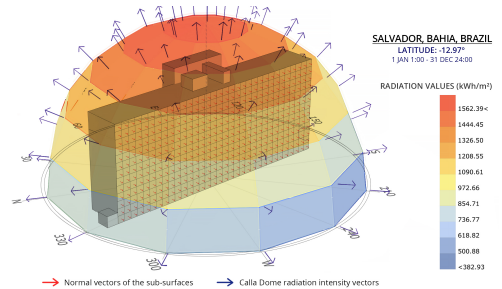

Figure 3

Case study geometry and Radiation Calla Dome models

After the facade form-finding process using Calla Dome model, the best genomes were submitted to the Radiation Simulation to evaluate the amount of radiation potential of each of the best facade formfound.

\section{Shape parameterization}

Into a three-dimensional isoparametric local reference domain $(u, v, w)$, which is in real values of cartesian space in units of meters, it was conceived the generative algorithm to model the facade shape surface based on NURBS and sinusoidal (sine function) curves. In both proposals, the shape parameterization is a topological generation through the definition of points, curves, and a surface, in this order. The points parameterize the geometry, controlling the NURBS surface shape, which, in turn, it is generated from a net of isocurves created from these same control points.

To contextualize the facade surface model with the building case study, a Boundary representation (B-rep) was modeled with boxes to represent its volume serving as a reference for the parametric facade modeling and as the context for the radiation simulations.

The NURBS curve models were parameterized from five points, of which three are inner control points. The local coordinate system origin position 
Figure 4

NURBS isocurve generation

Figure 5

Sine function

isocurves

generation

Figure 6

16 different initial parameters configuration for generating surfaces is in the vertical (u) and horizontal (v) origin domains, constructed on the building base model, represented by the number zero in Figure 4 . The horizontal domain represents the building length, 66.30 meters, and the vertical domain represents the building height, 26.20 meters, and within these domains, there are intervals subsets to determine the positions of the points sub-domains of each these five points, without overlapping. For each point, there is a domain value for the surface normal direction $\mathrm{w}$, ranging from 0.0 to $5.0 \mathrm{~m}$.
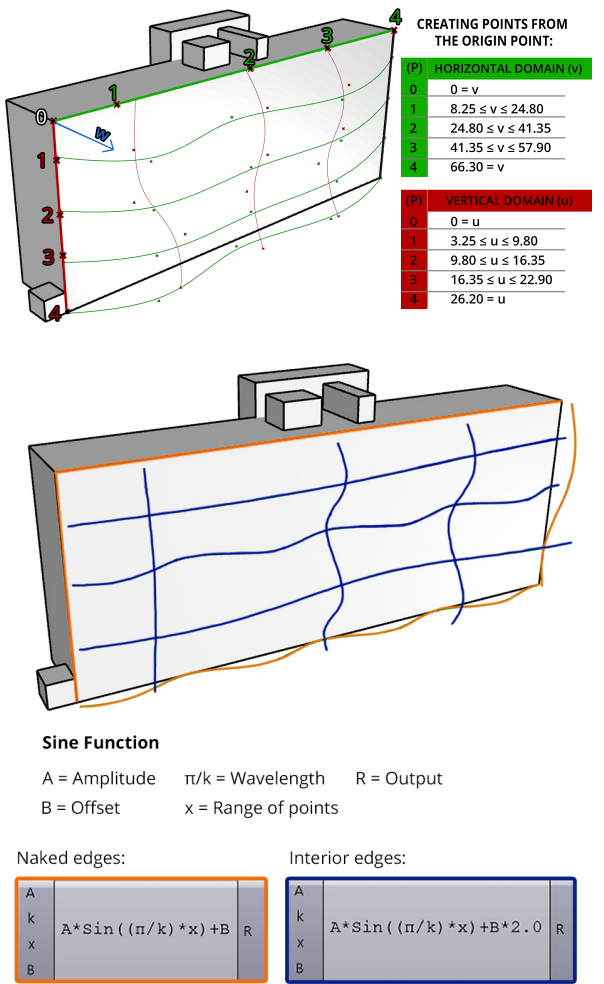

In the other hand, the Sine Function curve models were parameterized from the same reference, considering the end points of the curves within these domains, but the intermediates are the result of the other parameters belonging to the sine function. This set of internal points varies its positions through an axis in a local coordinate system and, being influenced by the parameters inside the sine function. With an interpolation of these points, it is created a series of undulating and wavelike isocurves, which its amplitude is independent for each isocurve, varying from $0.0 \mathrm{~m}$ to $2.0 \mathrm{~m}$.

Figure 5 shows these sine function parameters and how it changes according to the isocurve position. This multiplication factor 2 by the offset at the interior isocurves was defined so that there were no points generated inside the building model. Each sine curve is defined by the number of waves $k$ in the defined domain.

The generative process of surfaces of both proposals, from NURBS and sinusoidal isocurves, for the building southwest rectangular facade, was premised on fixing or releasing the edges named by A, B, C and D, as shown in Figure 6. Each capital letter represents a particular fixed edge in boundary conditions. As a proposition of this study, all instances have maintained the edge $A$ fixed offering a better sealing at the top of the facade.

The shape parameter control points position domains, in its lower and upper bounds setups, were taken as the initial conditions of parameters values, in which the lower bound starts from the minimum coordinate value of each control point position parameter domain and, in the other hand, upper bound starts from the maximum coordinate value. These limits are used as an initial genome in the GA process presented in the next section.

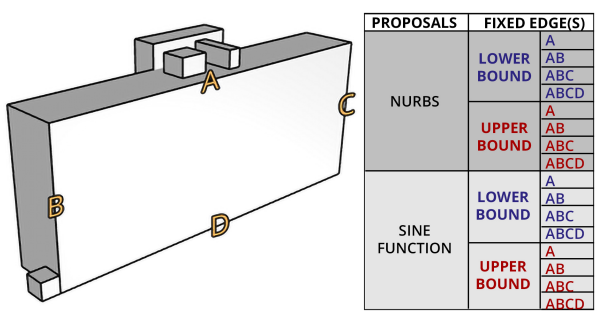


With these two generative curves proposals, the facade surface geometry model was given by the horizontal and vertical isocurves as input in a surface curve network algorithm. Since the surfaces obtained are curved, it needs to be subdivided to have more than one normal vector for representing their curvature directions. These surfaces divided into ten sub-surfaces in ( $u$ ) direction and twenty sub-surfaces in (v) direction, which from them can be gathered the normal vectors for calculating the angles between Calla Dome vectors and the normal vectors of these sub-surfaces, in order to be considered in the shape optimization generative algorithm.

\section{Facade form-finding}

By the iso-surface parameterization of the building facade, it was used the Radiation Calla Dome vectors and its radiation intensity values to interact with the sub-surfaces normal vectors.

For this task, it was composed of a Python script with two input data. The first one is the angles between the Calla Dome vectors and the normal vectors of the isoparametric sub-surfaces subset, represented by the variable 'angles'; The second one is the radiation values of Radiation Calla Dome associated to each Calla Dome vector, represented by the variable 'Rad'. These two-inputs are applied in the code to get an output given by the variable ' $F F^{\prime}$ ' which is the fitness function. This output divides the intensity of the Calla Dome radiation intensity by the angle between the Calla Dome vectors and the normal vectors of the sub-surfaces.

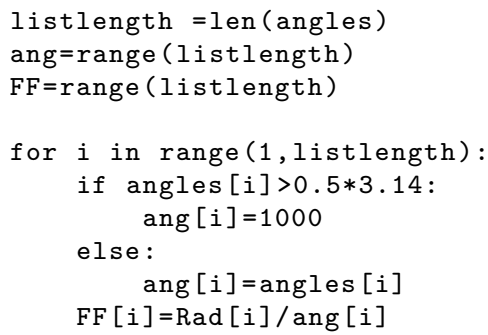

$\mathrm{FF}=\operatorname{sum}(\mathrm{FF})$
In the Python code definition, 'listlength' represents the length of the list of all angles between each Calla Dome vector and each of the surface normals for its evaluating; 'ang' is modified angle between the Calla Dome and the normal vectors of the sub-surfaces, being penalized by the value 1000 when the angle is superior to 90 degrees; and ' $F F^{\prime}$ ' represents the sum of all ratios. Therefore, each facade surface instance has this score made by the sum of all the ratios relating one to one vector between Calla Dome vectors and normal vectors of the sub-surfaces. The penalty upon the angles between vectors is necessary to do not consider Calla Dome vectors that would interact with the facade surface model interior side.

Maximizing fitness function score for optimizing through the GA environment Galapagos, it is expected to obtain shapes with optimum radiation incidence.

The Galapagos as an evolutionary solver consists of an algorithm that aims to find optimum solutions, inspired by Darwin theory (Floreano and Mattiussi 2008). The GA starts with the choice of the maximum number of stagnant generations as 50 , and the value of 50 was chosen. And, from that value is multiplied by an initial boost of 2 to promote the first generation. The individuals' evaluation is performed according to the defined fitness function, which the best individuals are those that reach close to that fitness values optimization objective. Even so, it is considered recombination and mutation between each one generation of individuals, regarding maintain of $10 \%$ of best individuals that can be carried over to the next generation and $75 \%$ for inbreeding factor.

Hence, it was also considered in GA, value parameters into their initial setup condition as lower and upper bounds as defined in the generation of isocurves. Consequently, the GA searches the best combination of these range values, maximizing the fitness value from Python's code output.

\section{PV facade preliminary design}

From the 16 NURBS surfaces generated through the form-finding process, meshes are obtained from the 
NURBS surface, in order to consider the edges of meshes as the facade support structure. And it turns into 16 triangle meshes (tri-mesh) and 16 quadrilateral meshes (quad-mesh), which through these meshes faces it was evaluated the distribution of the solar cells. The sizing of these mesh faces approaches the conventional photovoltaic panels sizes, which is about $1.0 \mathrm{~m}$ length and presents radiation simulation results values similar to a more accurate $0.50 \mathrm{~m}$ mesh discretization. However, if this edge size reduces too much, it could be found a problem of compatibility between the solar cell and facade mesh face shapes, thus fitting fewer cells. On the other hand, if it increases too much the mesh edge size, the surface curvature resolution is lost, as well as of the radiation incident on that generated NURBS surface.

Due to the most common application (D.G.S 2008), the sizing of solar cells is a square of $15 \mathrm{~cm} \mathrm{x}$ $15 \mathrm{~cm}$, and that it was arranged repeatedly in a rectangular array throughout the mesh faces.

Nevertheless, quad-meshes need to be planarized to make it possible to fabricate the structure and facade cladding using planar elements. And for this reason, the EvoluteTools plugin was used to perform the mesh planarization of the quad-meshes obtained, which consists of a mesh optimization algorithm. It was tested as base the generated 16 quadmeshes, for creating newer 16 quad-meshes, deviating minimally from the original shape, but with some mesh faces showing true planarity.

The ' $R$ ' coefficient in this study is defined, by the ratio between the total mesh area and the distributed photovoltaic cells cumulative total area distributed on the mesh faces. The solar radiation simulation results obtained for each generated facade solution were compared to the flat facade shape, in order to verify the effectiveness of considering curved shapes for it, in terms of gains of incident radiation or its photovoltaic potential.

\section{RESULTS}

In this section are presented a comparison between all 32 meshes instances against the original planar fa- cade shape in terms of incident radiation on the facade surface model. On the two chosen grid-type, the solar cells do not distribute with the same performances. Using Radiation Analysis algorithm, it was evaluated their total radiation $\left(\mathrm{kWh} / \mathrm{m}^{2}\right)$, mesh area $\left(\mathrm{m}^{2}\right)$, the sum of the area of distributed solar cells $\left(\mathrm{m}^{2}\right)$ and the ratio $\mathrm{R}$ between these two values, for analyzing their gains and losses in radiation potential according to the reference considering the flat facade shape.

Figure 7 presents the results of the radiation analysis for each type of isocurve, initial condition, and edge fixation, resulting in a vary incident radiation values at each point of the facade grid over the year. The evaluation of the radiation analysis between the tri-mesh and the quad-mesh showed to be visually equal through the false color gradient representing the intensity of the radiation per area, thus allowing to compare the results in more detail in Figure 9.

In these results, it can be seen that the sinusoid curves were more promising, obtaining the results with higher total radiation rates, comparing them with the surfaces generated through the NURBS curves, taking as reference the vertical flat facade shape. The surfaces tend to have their faces on top more horizontally tilted, optimizing the solar incidence on this region in this case study, represented by the red color. The blue areas represent regions of the façade where they are more recessed, casting shading on them. The yellow areas prevail over the result, ranging from $741.32 \mathrm{kWh} / \mathrm{m}^{2}$ to 988.42 $\mathrm{kWh} / \mathrm{m}^{2}$.

Figures 8 shows the distributions of the photovoltaic cell on the different faces of tri-mesh and quad-mesh, respectively. Solar cells do not fill all the mesh faces, due to their size and shape, resulting in gaps in faces grid modules. 

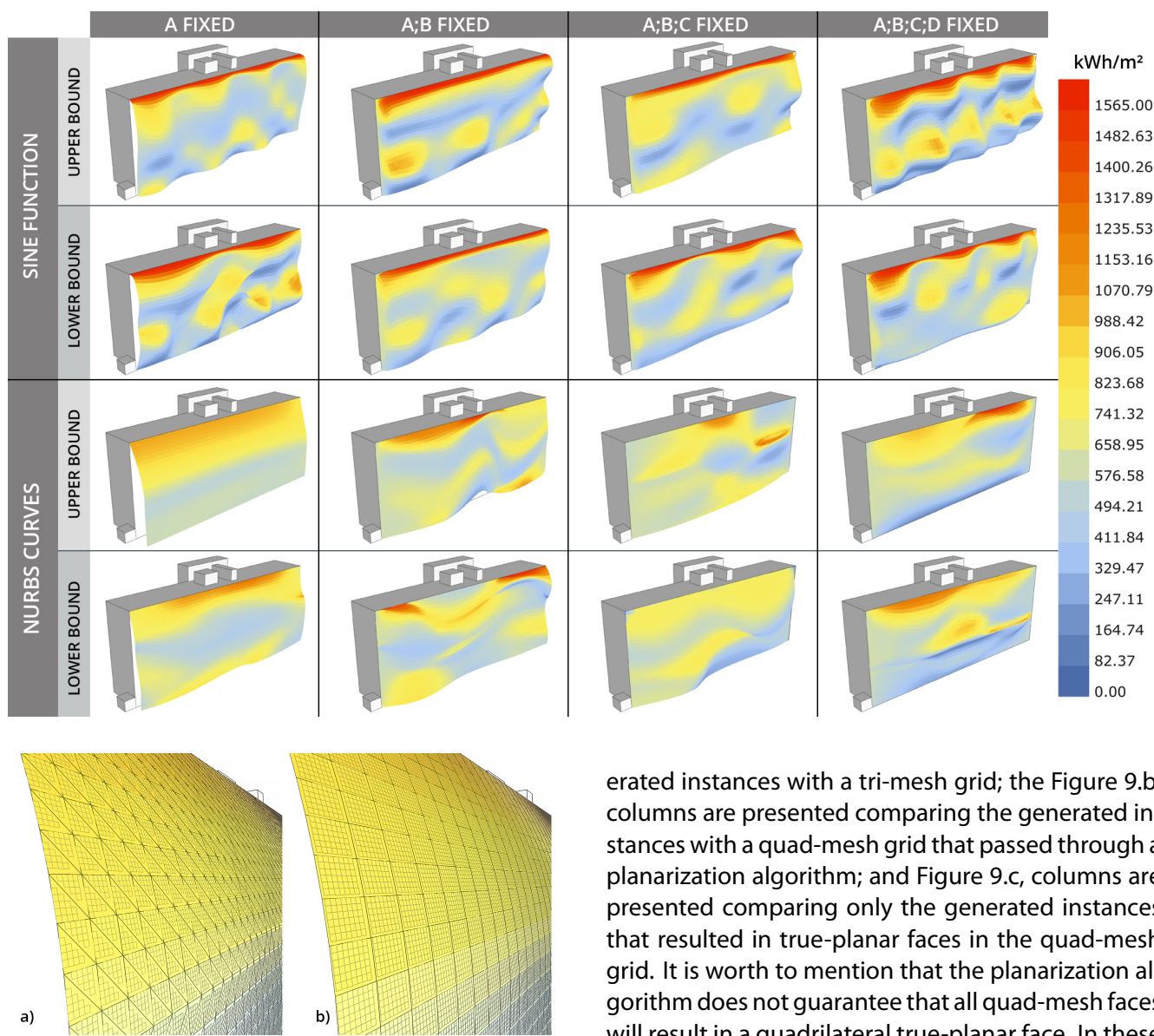

The results obtained from the flat facade shape as the total radiation was $928,820,263.00 \mathrm{KWh}$, the mesh area of $1,736.9 \mathrm{~m}^{2}$, the cumulative area of the distributed solar cells $1,389.96 \mathrm{~m}^{2}$. To show the equivalent results brought by the curved shapes obtained, a bar-plot was constructed to compare the results with this flat shape reference. Figure 9 expresses in percentage inside a bar-plot, the solar potential gains, and losses of the generated mesh and faces of PV cells against the flat facade shape. In the Figure 9.a, columns are presented comparing the gen-
Figure 7

Radiation analysis results

erated instances with a tri-mesh grid; the Figure 9.b, columns are presented comparing the generated instances with a quad-mesh grid that passed through a planarization algorithm; and Figure 9.c, columns are presented comparing only the generated instances that resulted in true-planar faces in the quad-mesh grid. It is worth to mention that the planarization algorithm does not guarantee that all quad-mesh faces will result in a quadrilateral true-planar face. In these quad-meshes instances, all the 16 cases could not have all the mesh faces planarized.

In all tri-mesh cases, they had gained on the mesh faces in relation to the flat proposal, comparing the amount of radiation gathered by the mesh faces. However, they lost in all cases of PV cells faces distribution, except for sinusoid isocurves ABCD fixed (upper bound as Initial Condition model parameter setup), which had an increase of $4 \%$ of gain in total radiation in comparison to the reference.
Figure 8

PV cells distribution over the supporting grid: a) Tri-mesh grid; b) Quad-mesh grid 
Figure 9

Solar potential gains and losses against the flat facade shape: a) Tri-mesh case; b) Quad-mesh case (all); c) Quad-mesh case (true planar faces only) a)

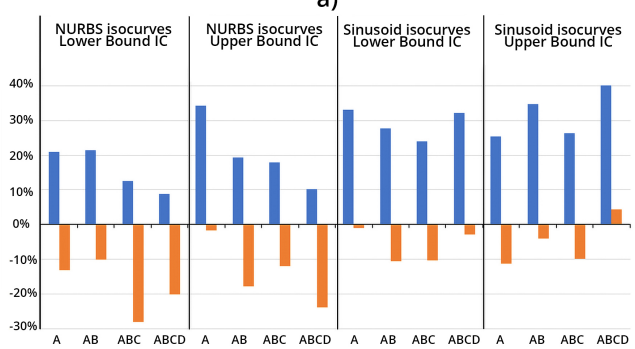

b)

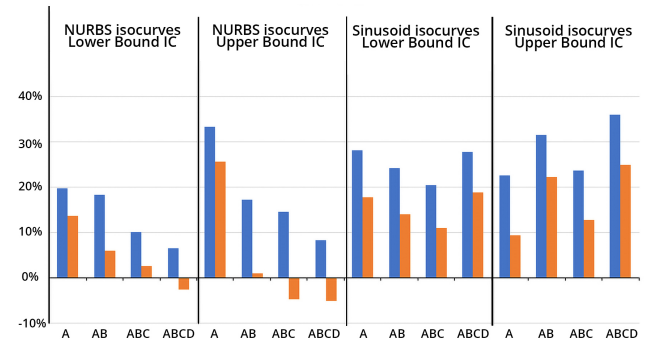

C)

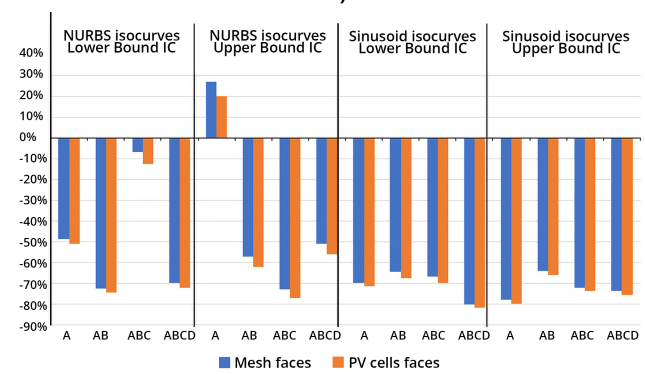

In most quad-mesh cases they had a good gain compared to the flat surface, which is expected once the curved shape has more area than a flat shape. An increase of up to $35 \%$ in radiation gain taking the mesh faces areas, as for example in sinusoid isocurves $A B C D$ fixed (upper bound as IC). But they also presented losses in some cases. NURBS isocurves $A B C D$ fixed (lower bound as IC), NURBS isocurves $A B C, A B C D$ fixed (upper bound as IC) had up to $-5 \%$ loss in radiation when the $P V$ cell faces distribution is considered in comparison to the flat reference.

In most cases, quad-mesh with true-planar faces only had a significant loss in all the cases in comparison to the flat surface. A decrease of up to $80 \%$, as for example in sinusoid isocurves $A B C D$ fixed (lower bound as IC). Only one case had a gain: the NURBS isocurves A fixed (upper bound as IC) typology had to increase in comparison to the flat surface. In both cases, mesh faces and distributed PV cells faces showed results of up to $25 \%$ in gain compared to the vertical flat facade surface.

\section{DISCUSSION AND CONCLUSION}

The Radiation Calla Dome and Radiation Analysis algorithms were essential to visualize this environmental analysis through 3D representations. In this study, the first one was used for the form-finding process, while the second one for analyzing the radiation performance obtaining specific values over the generated meshes. Thus, it was possible to compare the values generated between them and the flat facade reference.

Besides that, there are issues about this study that interacts the process to obtain 3D model shapes, as for example, the building has already the geometry defined, and at the other hand, due to the school parking lot position, there is no direct context shading promotion, successfully aiding the incident radiation on the studied facade surface.

The facade solar PV system intersects direct solar irradiation differently from a horizontal or an optimally tilted system, during the day and throughout the year. By means of the GA association with different variations of parameters in the curve generations and boundary conditions, it was possible to generate several solutions with curved forms on the facade. Depending on the type of the curve leads to a great amount of self-shadowing, obstructing the radiation from falling more intensely on the facade.

Another point at issue is the difference between the geometric model and possible model to be built. Despite the planar faces of tri-mesh, its configuration is not very compatible with the shape of the solar cell, which does not allow distribution over a great amount of cumulative area. While quad-mesh 
has higher radiation values and greater compatibility with quadrilateral solar cells, on the other hand, not all quadrilateral mesh faces can be planarized because of the surface generation method or its boundary conditions. Only one instance, NURBS isocurves fixed in ' $A$ ' with initial conditional in upper bound, obtained a result superior to the flat facade shape, considering these two isocurve surface topologies.

This study promoted a new possibility of generating preliminary photovoltaic facades shapes with a more curved design and optimized in relation to incident solar radiation. It is worth to mention that it is necessary to complement this investigation with the consideration of the other factors that the facade design demands. Besides, through parametric modeling, the insertion of a weather database associated with generative algorithms promotes more assertive results and can be applied to any other facade study anywhere in the world.

\section{ACKNOWLEDGMENTS}

The authors would like to thank the CNPq Research Brazilian agency to provide support to this research by the Universal MCTI/CNPq No01/2016 call, under the project number $400437 / 2016-3$. And also thank the plugin Ladybug owners to offer its availability to the scientific research use.

\section{REFERENCES}

Ashdown, I 2004 'Modeling Daylight for Interior Environments', Proceedings of the Annual Conference IESANZ

Floreano, D and Mattiussi, C 2008, Bio-inspired Artificial Intelligence: Theories, Methods, and Technologies, The MIT Press, Cambridge

Freitas, SRT 2018, Photovoltaic Potential in Building Façades, Ph.D. Thesis, Ciências ULisboa

Kalogirou, S 2009, Solar Energy Engineering: Processes and Systems, Elsevier, Burlington

Martino, JA 2015, Algoritmos Evolutivos como Método para Desenvolvimento de Projetos de Arquitetura Ph.D. Thesis, UNICAMP

MR, Sadeguipour and Pak, M 2013 'Ladybug: A parametric Environmental Plugin for Grasshopper to Help Designers Create an Environmentally-conscious Design', Proceedings of the 13th International IBPSA,

$$
\text { Lyon }
$$

Probst, MCM and Roecker, C 2012, Solar Energy Systems in Architecture: Integration Criteria and Guidelines, Solar energy \& Architecture International Energy Agency

Robinson, D and Stone, A 2004 'Irradiation Modelling Made Simple: The Cumulative Sky Approach and its Applications', Proceedings of the 21st Conference on Passive and Low Energy Architecture, Eindhoven

Sanna, A, Achenza, M and Desogus, G 2015, Guidelines on Building Integration of Photovoltaic in the Mediterranean Area, Foster in Med, Cagliari

Sarbu, I and Sebarchievici, C 2017, Solar Heating and Cooling Systems: Fundamentals, Experiments and Applications, Elsevier, London

Silva, H, Sparn, JOW and Vieira, RG 2016, 'Development? Thinking the Future Through an Urban-natural Perspective', Nova Economia, 26, pp. 1157-1186

The German Energy Society, DGS 2008, Planning and Installing Photovoltaic Systems: A Guide for Installers, Architects and Engineers, Earthscan Publications Ltd, Berlin

Tregenza, PR and Waters, IM 1983, 'Daylight Coefficients', Lighting Research \& Technology, 15(2), pp. 65-71

[1] http://www.ren21.net/wp-content/uploads/2018/0

6/17-8652_GSR2018_FullReport_web_final_.pdf

[2] https://www.iea.org/media/training/eetw2016/buil dings/A.2_Energy_efficiency_potential.pdf

[3] http://www.iea-pvps.org/fileadmin/dam/public/rep ort/statistics/2018_iea-pvps_report_2018.pdf

[4] https://globalsolaratlas.info/?c=5.707184,-1.078204, $3 \& s=-12.9822,-38.4813 \& \mathrm{e}=1$

[5] https://www.radiance-online.org/about/detailed-de scription.html\#section-0 